\title{
Calculation of locomotive traction force in transient rolling contact
}

\author{
P. Voltr ${ }^{a, *}$

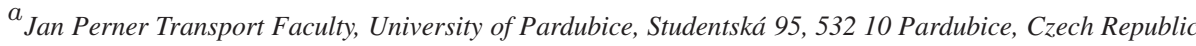 \\ Received 12 December 2016; accepted 7 February 2017
}

\begin{abstract}
To represent the wheel-rail contact in numerical simulations of rail vehicles, simplified models (Fastsim, Polách etc.) are usually employed. These models are designed for steady rolling only, which is perfectly suitable in many cases. However, it is shown to be limiting for simulations at very low vehicle speeds, and therefore it does not actually allow simulation of vehicle running at arbitrarily variable speed. The simplified model of transient rolling, which involves calculation of the stress distribution in the discretised contact area, overcomes this disadvantage but might be unnecessarily complex for more simple simulations. In this paper, an approximative creep force computation method for transient rolling is presented. Its purpose is not to study the transient phenomena themselves but provide a simple and readily available way to prevent incorrect results of the numerical simulation when the vehicle speed approaches zero. The proper function of the proposed method is demonstrated by a simulation of start-up and interrupted sliding of a four-axle locomotive.
\end{abstract}

(C) 2017 University of West Bohemia. All rights reserved.

Keywords: wheel-rail contact, adhesion, creepage, rolling, transient rolling

\section{Introduction}

In modern research and development of railway vehicles, investigation of dynamic behaviour of a vehicle or its subsystem is a frequent task. This requires modelling of the vehicle-track dynamical system, usually involving a multi-body system of masses, linkages and force elements $[2,6,9,16]$. The force elements include springs, dampers and similar components, but also - which is of great importance - the wheel-rail contact elements.

The wheel-rail contact model represents the principal behaviour of the interface of wheel and rail, namely transmission of tangential forces in the presence of creepage. Creepage (or creep) is a phenomenon consisting in the difference of the wheel movement from pure kinematic rolling. Creepage does not directly imply full sliding, since it may be (partially) compensated by tangential deformation of the bodies of wheel and rail. The term "creepage" also denotes a physical quantity describing the creep phenomenon $[6,9,12]$.

In dynamics of railway vehicles, the task of wheel-rail contact modelling is to describe the contact of wheel and rail in terms of dependence of tangential forces on creepages [12]. The wheel-rail contact subsystem in vehicle dynamics simulations accepts the creepages (or, more generally, the motion of the wheel) as input, and provides the tangential forces as output. Various contact models have been developed over the years, with various simplifying assumptions that make the solution less accurate but reduce the computation time. For overview of contact models for vehicle simulations and comparison of their accuracy, see e.g. [17].

*Corresponding author. Tel.: +420 465038 520, e-mail: petr.voltr@upce.cz. https://doi.org/10.24132/acm.2017.335 
One of the common assumptions of wheel-rail contact models is that the wheel is in the mode of steady rolling (steady state of rolling with creepage). It will be explained that this is perfectly acceptable in most cases but there are other cases where the steady rolling assumption means a serious problem. The present study is focused on the phenomenon of transient rolling of a railway wheel and deals with the question how to include it in the vehicle simulations in a simple way.

\section{Short overview of the wheel-rail contact problem}

Consider a planar view of a rigid wheel supported by a rigid rail (Fig. 1a). The wheel rotates about its axis with angular velocity $\omega$ and, at the same time, moves forward with linear velocity $v$. In pure rolling, the relation $v=\omega r$ is satisfied (with $r$ the radius of the wheel). On the contrary, a difference velocity exists in rolling with creepage,

$$
w=\omega r-v
$$

which is termed the creep velocity and quantifies the creep effect. A dimensionless quantity is also available: this is called simply creepage and is expressed as

$$
s=\frac{w}{v_{\mathrm{r}}}=\frac{\omega r-v}{v_{\mathrm{r}}}
$$

where $v_{\mathrm{r}}$ is a reference velocity, selected either as $v$, or $\omega r$, or their arithmetic mean. Kinematic analysis of a wheel in three dimensions clarifies that besides the longitudinal creep velocity and creepage $\left(w_{x}, s_{x}\right)$, there is also the lateral creep velocity and creepage $w_{y}, s_{y}$. In addition, angular movement of the wheel about the contact normal is possible, which can occur even without the presence of wheelset yaw movement if the wheel tread is conical. This movement is called spin in wheel-rail contact theory and is quantified by the angular velocity of spin $\omega_{z}$ and the relative spin creepage $\phi=\omega_{z} / v_{\mathrm{r}}$. For more detailed explanation, see e.g. [4,12,13].

It has been said that, in contrary to the previous assumption, the wheel and rail are not rigid. The primary consequence of this is that a contact area is formed around the geometrical point of contact under the action of normal force $N$. Finding the shape and size of the contact area, as well as the distribution of normal stress or pressure $p(x, y)$ over this area, is the task of the normal contact solution. The theory of Hertz (originally published in [8], also explained e.g. in [13]; see Fig. 1b) is commonly used in the normal contact problem.

(a)

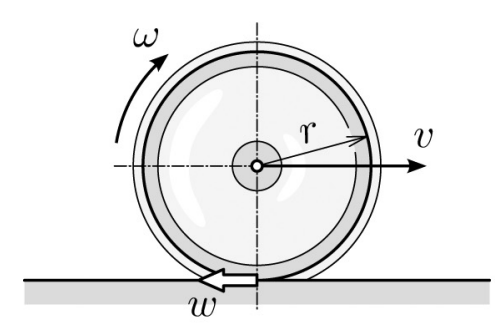

(b)

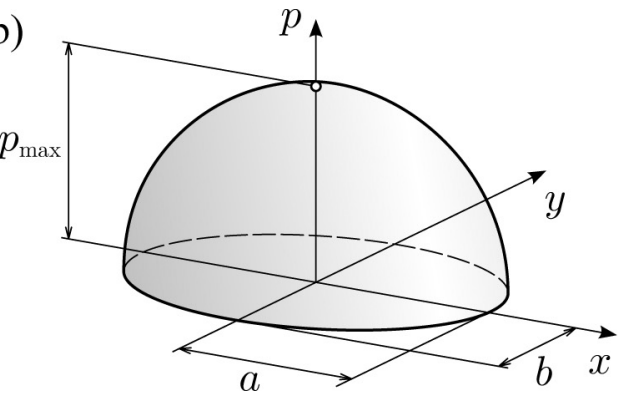

(c)

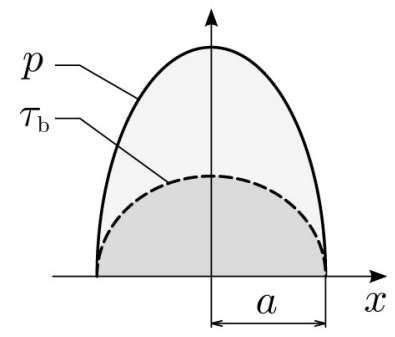

Fig. 1. Illustration to the wheel-rail contact problem: (a) movement of a wheel in a planar model, (b) distribution of pressure in Hertzian contact with $a, b$ semiaxes of the contact ellipse, (c) longitudinal section of this distribution together with traction bound $\tau_{\mathrm{b}}$ 
The compliance of the wheel and rail bodies also means that besides the normal deformation (compression), tangential deformation (shear) may take place, which also means that the creep velocity as defined above is not generally equal to the actual velocity of sliding between the surfaces. The shear deformation occurs under the action of tangential stress (traction, $\tau$ ) at the interface, and this relation is governed by the principle of elasticity. One may distinguish so-called exact theory, dealing with a full elastic continuum model of the bodies, and simplified theory, reducing the bodies to rigid bases with flexible bristles at their interface (also called the brush model, analogy to the Winkler bedding), illustrated in Fig. 2, [6, 12, 13].

(a)

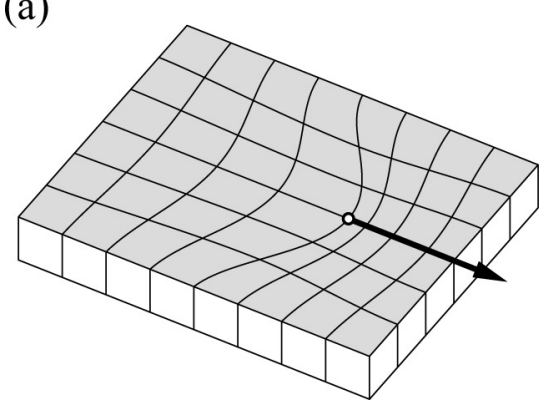

(b)

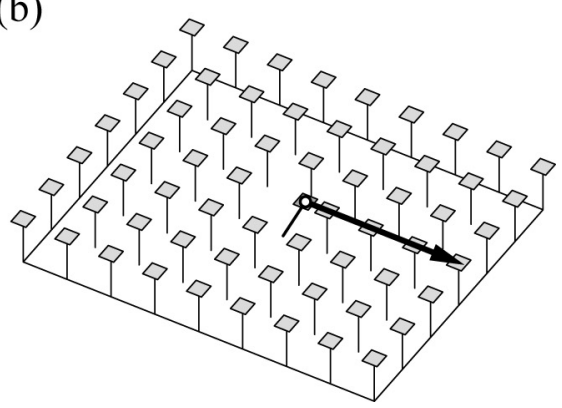

Fig. 2. The effect of a tangential loading in a surface point (a) on a full elastic material model, used in "exact" contact theories, and (b) on a brush model, on which "simplified" theories are based

The traction is limited by the traction bound $\tau_{\mathrm{b}}$, representing the application of the friction law on the contact area. The traction bound is a distribution of maximum possible magnitude of tangential stress,

$$
\tau_{\mathrm{b}}(x, y)=f \cdot p(x, y),
$$

with the coefficient of friction $f$ (see Fig. 1c). In the parts where $\tau<\tau_{\mathrm{b}}$, static friction is not overcome and the surfaces stick to each other - this is called the zone of adhesion. In other parts of the contact area with $\tau=\tau_{\mathrm{b}}$, sliding is present (zone of sliding, or slip).

In creep force calculations, the traction distribution is found based on the creepages and then integrated to obtain the resulting force effects: longitudinal force $T_{x}$, lateral force $T_{y}$ and spin moment $M_{z}$.

\section{Steady and transient rolling}

As the wheel moves forward on the rail, particles of surfaces of the wheel and rail enter the contact area at its leading edge. They proceed through the contact area, carrying their stress and strain with them, and finally leave the contact at the trailing edge.

One of the commonly accepted simplifications in wheel-rail contact models is steady rolling, which means rolling without change in conditions that influence the traction in the wheel-rail contact, particularly creepage. In steady rolling, creepage is constant in time, therefore traction in all points (with the material flowing through the contact zone) is governed by the same conditions. Consider a simplified material model and pure longitudinal creepage: under these conditions, distribution of tangential stress starts with linear increase at the leading edge and is limited by the traction bound in the rear part. The slope is proportional to creepage $s$ with the relation

$$
\frac{\mathrm{d} \tau}{\mathrm{d} x}=\tan \gamma=K \cdot s
$$




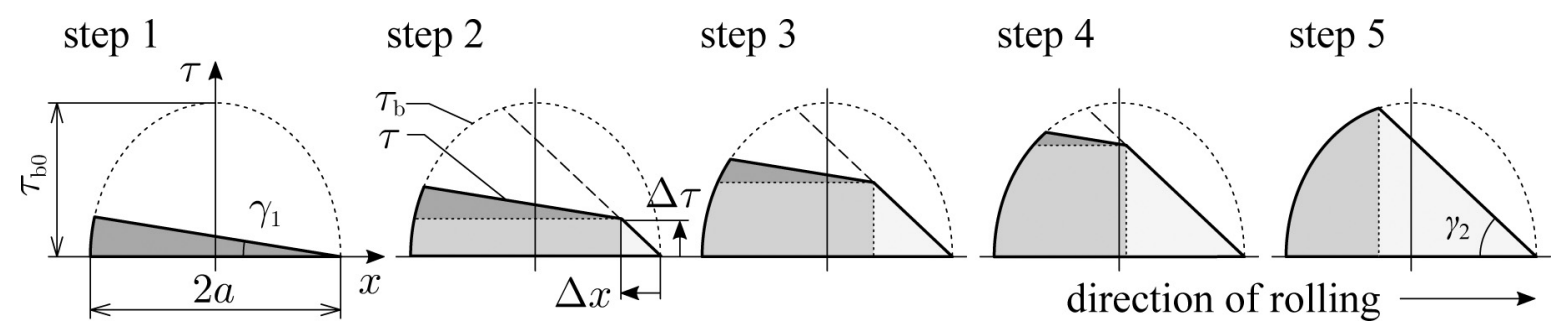

Fig. 3. Longitudinal section through the traction distribution $\tau$ and traction bound $\tau_{\mathrm{b}}$ in a transient rolling process with prescribed stepwise increase of creepage

where $K\left[\mathrm{~N} / \mathrm{m}^{3}\right]$ is a parameter expressing the tangential stiffness of the surface layers of wheel and rail. If the contact is Hertzian and no spin is present, the traction distribution may be integrated analytically and a simple formula for the creep-force relationship ("adhesion characteristic") may be given $[5,11,15]$.

Transient rolling is a mode of wheel motion in which creepage changes in time. Considering the flow of particles through the contact area, this results in a deformation/stress state that depends on the history of creepage. Fig. 3 shows how the traction distribution changes in response to a step increase in creepage. In each displayed step, the wheel advances by $\Delta x$ and traction increases by $\Delta \tau$. For similar diagrams in various scenarios, see e.g. $[10,13,19]$.

With the nature of transient rolling explained, the duration of the transient phenomenon (response to a step input variation) can be guessed: the transition will finish when the wheel advances by the contact length, at the latest. ${ }^{1}$ The contact length might be, for instance, $15 \mathrm{~mm}$; at the vehicle speed of $40 \mathrm{~km} / \mathrm{h}$, the wheel makes this distance in $1.4 \mathrm{~ms}$. Indeed, the transient phenomenon is very short and can be neglected in investigation of vehicle running behaviour, which is normally focused on speeds higher than that.

The duration of the transient phenomenon is longer for lower vehicle speeds, which makes the assumption of steady rolling less suitable. In the very first movement of the wheelset from zero speed, the results calculated using a steady rolling model are completely wrong. This is a problem for simulation of low vehicle speeds, which may be of interest particularly in locomotive traction with the tractive effort most significantly limited by adhesion at low speed. Also, in consequence, the steady rolling approach does not allow simulation with arbitrarily variable vehicle speed.

To allow inclusion of the transient rolling in vehicle dynamics simulations, the simplified model of transient rolling (STR) was devised $[18,19]$. Its assumptions are the same as those of Fastsim, except for the steady state of rolling. The contact area is discretised in a rectangular grid. In each computational step, new traction distribution $\tau(x, y)$ is calculated from the previous one and then integrated. This method is essentially suitable for all components of creepage but has been algorithmized only for longitudinal creepage so far. In this case, the basic formulae for stress calculation are

$$
\tau_{\mathrm{a}}(x, y, t)=\tau(x-\Delta x, y, t-\Delta t)+\Delta \tau=\tau(x-v \Delta t, y, t-\Delta t)-K w \Delta t
$$

for the no-slip (adhesion) solution $\tau_{\mathrm{a}}$, and

$$
\tau(x, y, t)= \begin{cases}\tau_{\mathrm{a}}(x, y, t) & \text { for }\left|\tau_{\mathrm{a}}(x, y, t)\right|<\tau_{\mathrm{m}}(x, y, t) \\ \tau_{\mathrm{b}}(x, y, t) \cdot \operatorname{sgn} \tau_{\mathrm{a}}(x, y, t) & \text { for }\left|\tau_{\mathrm{a}}(x, y, t)\right| \geq \tau_{\mathrm{m}}(x, y, t)\end{cases}
$$

to apply the limitation by the traction bound and obtain final traction distribution $\tau$.

\footnotetext{
${ }^{1}$ The transition is somewhat slower in exact theory (elastic continuum) where the particles outside of the contact area are not free from stress and strain, although the surface stress is, of course, zero.
} 
The STR might be unnecessarily complicated for simple applications where the purpose is not to study the transient phenomena themselves but it is only needed to avoid incorrect behaviour for very low speed or similar cases. In the next section, a more simple approach designed for locomotive traction studies is proposed, which anyone could easily implement in simulation.

\section{The new proposed method}

\subsection{Basic principle}

The objective is to develop a method of calculation of tangential force in transient rolling contact based on a steady-state model (e.g. Polách [15]) rather than on numerical calculation of the traction distribution in the discretized contact area.

Consider a wheel-rail contact model taken out of the vehicle-track dynamic system, thus representing a block with creepages as input and forces as output. Let us confine to pure longitudinal creepage and observe the response of such a block to step increase of creepage in time $t=0$ :

$$
s(t)= \begin{cases}s_{1} & \text { for } t<0 \\ s_{2} & \text { for } t \geq 0\end{cases}
$$

Any steady-state contact model produces a corresponding jump in the tangential force from $T_{1}$ to $T_{2}$. On the other hand, a continuous increase is observed in transient rolling contact. This increase starts non-smoothly with the greatest slope in the beginning and converges to $T_{2}$ (Fig. 4). This resembles the response of an exponential smoothing filter

$$
x_{\mathrm{f}}^{(i)}=F \cdot x^{(i)}+(1-F) \cdot x_{\mathrm{f}}^{(i-1)},
$$

where $x$ represents the input signal, $x_{\mathrm{f}}$ is the filtered output, $i$ is the index of the current instant and $F$ is the constant of the filter. This algorithm combines the new input value with the previous output value, weighed by the constant $F$, to produce the new output.
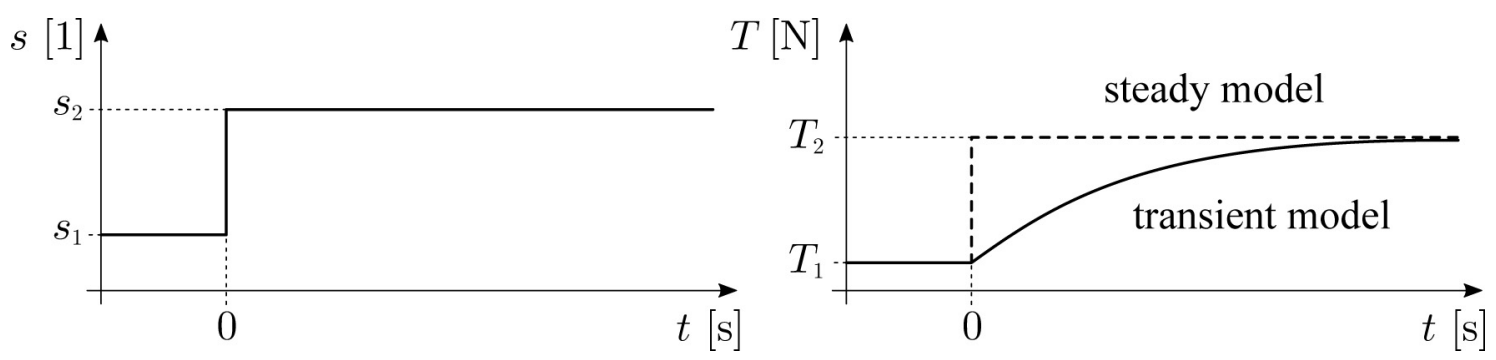

Fig. 4. Sketch of the responses of a steady-state rolling model and a transient model to a step increase in creepage

For the application in transient rolling, physical meaning is also not altogether absent from this approach: in each computation step of the transient rolling process, the resulting tangential force depends in part $(F)$ on the traction in the points that have just entered the contact area, and in the other part $(1-F)$ on the traction in the points that already were in contact before. This suggests to use the principle of exponential filter in the transient rolling model: a steady-state formula will be used, but the filtering will be incorporated at some stage of the calculation process. 


\subsection{Formulae of the model}

To transfer the principle in equation (8) to the creep force calculation, two questions must be answered. First, how to choose the value of $F$. Second, which quantity will be chosen to be subjected to the filtering.

It follows from the previous section that filter constant $F$ must depend on the distance travelled in the current computation step $\Delta x$. For $\Delta x>2 a$ (length of the contact area), it is certain that no points from the previous step remain in the contact area and there is new steady traction distribution in the contact area $-F$ must be equal to 1 . For $\Delta x=0$ (no advance in the current step), one might think that no new points enter the contact zone and $F$ should be zero. This approach would, however, fail in the case of traction increase without the wheel moving forward; and correct behaviour in such limit cases is the main requirement on the model.

To avoid these problems, another quantity will be used in the formula for $F$, in addition to distance $\Delta x$ : traction increase $\Delta \tau$. The formula must produce

- $F=0$ if nothing changes, i.e. $\Delta x=0$ and $\Delta \tau=0$,

- $F=1$ if the previous traction distribution is all lost either by the points leaving the contact area, $\Delta x \geq 2 a$, or the traction increased up to or beyond the traction bound maximum, $\Delta \tau \geq \tau_{\mathrm{b} 0}=\max \left(\tau_{\mathrm{b}}(x, y)\right)$

with smooth transition between these states. The following formula for the filter factor is proposed to meet the requirements:

$$
F=\min \left(\sqrt{\left(\frac{\Delta x}{2 a}\right)^{2}+\left(\frac{\Delta \tau}{\tau_{\mathrm{b} 0}}\right)^{2}} ; 1\right) .
$$

The value $\Delta \tau$ (element of traction increase in no-slip condition) is available in the simulationbased on the principle of the brush model with traction proportional to deformation $[11,15]$, it may be expressed as

$$
\Delta \tau=K \cdot \Delta x_{s} \approx K w \cdot \Delta t,
$$

where $\Delta x_{s}$ dentoes the element of creep motion in the current computation step.

Further, the quantity that will be subjected to filtering is searched. Originally, it appeared suitable to filter creepage $s$ before sending it as input to a steady-state creep force law. The filtering could then be interpreted as finding the steady-state traction distribution that results in the same total force as the present transient distribution. Nevertheless, this option resulted in serious problems in high creepages. The use of angle $\gamma=\arctan (K \cdot s)$, which reduces the creepage range of $\pm \infty$ to $\pm \pi / 2$, removed some of the problems. However still it proved best to apply the filter to the output of the steady state model-tangential force or coefficient of adhesion:

$$
x \equiv T \text { or } x \equiv \mu \text {. }
$$

Let us therefore call this approach the creep force filtering method (CFFM).

\subsection{Implementation}

The way how the above statements and formulae are embodied in the numeric solution is explained in the following steps. They describe the transient contact modelling in a dynamic simulation of locomotive traction phenomena, therefore with longitudinal creepage only. The normal contact is Hertzian; normal force $N$ and therefore the dimensions of the contact area can change with time. 
1. Obtain the quantities describing the wheel movement (translation, rotation) from the kinematics unit. This may be given as elements $\Delta x_{\mathrm{w}}$ and $\Delta \phi_{\mathrm{w}}$ indicating how the position of the wheel changed during the time step.

2. Calculate creep (difference) element $\Delta x_{s}=\Delta x_{\mathrm{w}}-\Delta \phi_{\mathrm{w}} r$ and average motion element $\Delta x=\left(\Delta x_{\mathrm{w}}+\Delta \phi_{\mathrm{w}} r\right) / 2$.

3. Calculate creepage $s=w / v=\Delta x_{s} / \Delta x$.

4. Solve the normal problem to find the parameters $a, b, p_{\max }$. Calculate coefficient of friction $f$ if a velocity-dependent friction model is used. Also prepare tangential stiffness $K$ :

$$
K=\frac{3 G C_{11}}{8 a}
$$

where $G$ is shear modulus, $C_{11}$ Kalker's coefficient for longitudinal creep force [15].

5. Calculate creep force $T$ for creepage $s$ using the steady-state Freibauer/Polách model $[5,15]$.

6. Find the value of filtering factor $F$, equation (9), with $\Delta x$ found in the second step and

$$
\Delta \tau=K \cdot \Delta x_{s}
$$

7. Apply the filter (8) to creep force $T$ calculated in step 5. Use the filtered value as output and store it also for the use in filtering in the next time instant.

Fig. 5 shows the block diagram of the process that also makes clear the difference from standard steady-rolling solution.

(a)

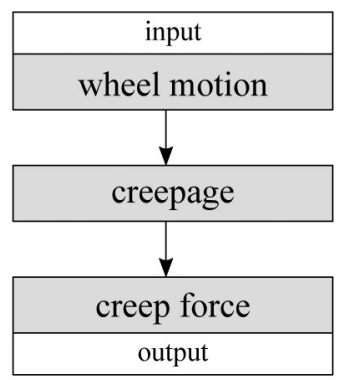

(b)

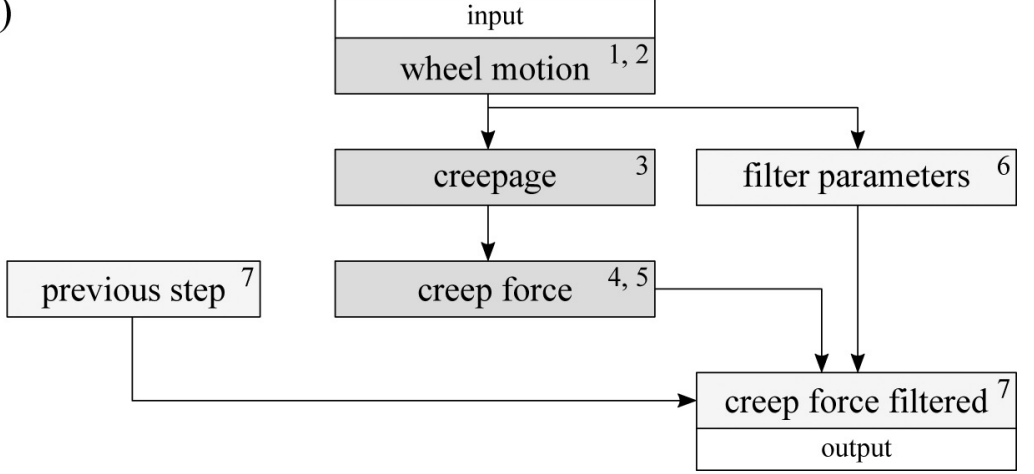

Fig. 5. The procedure of contact solution for (a) steady rolling, (b) transient rolling with creep force filtering; the numbers refer to the list in the text of Section 4.3

\section{Testing of the method}

\subsection{Quasi-static testing}

"Quasi-static testing" refers to testing of the response of the transient wheel-rail contact model to an input sequence of creepages. This sequence may also include variability of rolling velocity, contact size and pressure etc., but only the simplest case of only longitudinal creepage varying according to (7) and Fig. 4 is dealt with here. The normal contact is Hertzian. 


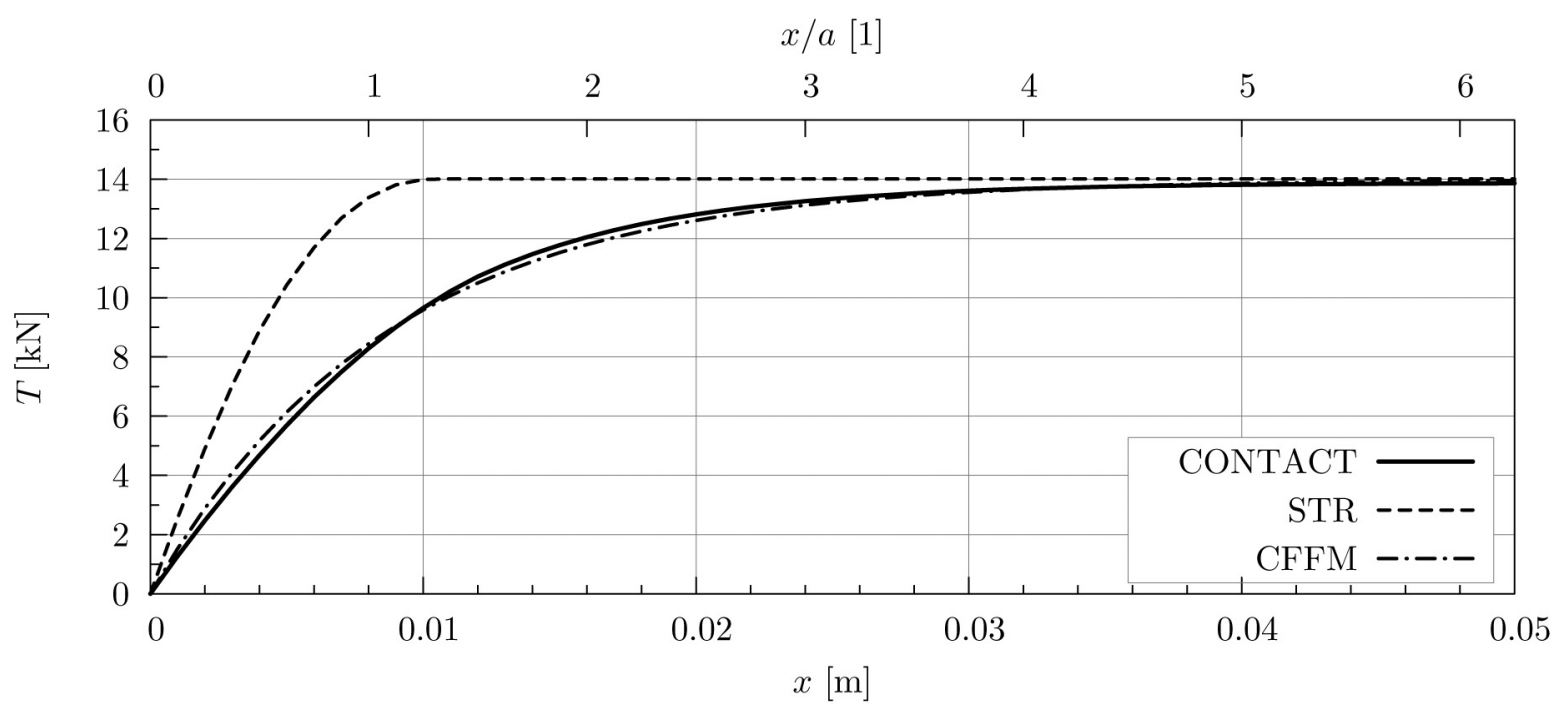

Fig. 6. Comparison of the models in a transient rolling scenario with $s_{1}=0, s_{2}=0.001$ : CONTACT, STR and the proposed method CFFM

The presented example is characterized by the parameters: contact area semi-axes $a=8 \mathrm{~mm}$, $b=6 \mathrm{~mm}$, maximum Hertzian pressure $p_{\max }=1000 \mathrm{MPa}$, Young's modulus $E=210 \mathrm{GPa}$, Poisson ratio $\nu=0.27$, coefficient of friction $f=0.2$, tangential stiffness of the brush model $K=17.87 \times 10^{12} \mathrm{~N} \mathrm{~m}^{-3}$. The values of creepage are $s_{1}=0$ and $s_{2}=0.001$.

Three transient contact models are compared: the exact theory of Kalker (algorithm CONTACT, implemented in the software available from [3]), STR and the proposed method CFFM.

In the first place, the difference between the exact solution and STR can be seen in Fig. 6. This difference is caused by two factors:

1. The simplifying assumption that steady-state stiffness/flexibility of the brush model can be used in transient rolling, too - this influences the initial slope of the curve. For the problem of transient rolling flexibilities, see [1,7].

2. The nature of the brush model in which no previous state influences the current state after the relevant particles leave the contact area - this causes the creep force to converge in finite time rather than to approach the steady value asymptotically.

This property of the STR has been known before, but it is not viewed as a critical disadvantage. This scenario - creepage jump from zero to $0.1 \%$ — constitutes a very rapid change of input that does not normally occur in dynamic simulation of vehicles. In a more realistic sequence of startup of a vehicle, the difference between STR and the exact theory was much smaller.

Nevertheless, the filtering method — although conceived as a very simple and approximate one - appears to give particularly good results in this test scenario, as it agrees with the result of the exact theory remarkably.

\subsection{Dynamic testing}

In "dynamic" testing, the wheel-rail contact model is implemented in a numerical simulation of dynamical behaviour of a four-axle locomotive. The model is a planar one, with the lateral coordinate omitted. The mechanical system of vehicle and track is a multi-body system with 30 degrees of freedom (Fig. 7). The force elements include linear springs and viscous dampers, 


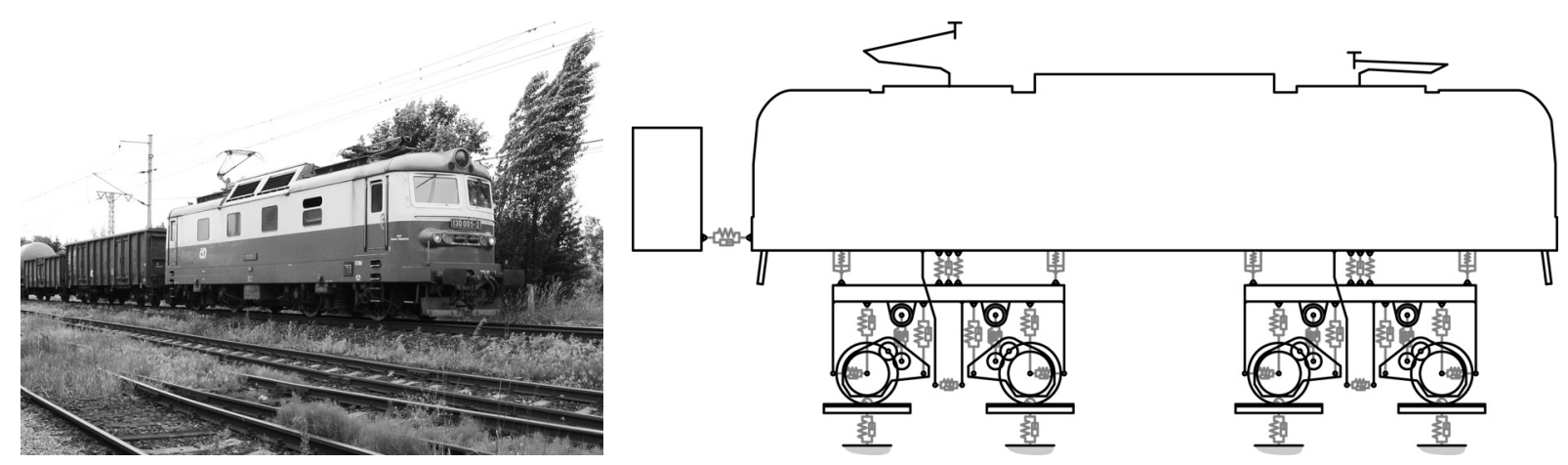

Fig. 7. The locomotive Škoda 79E and its mechanic model; the hauled vehicles are represented by a single body

nonlinear model of the electric traction circuit (for torque of the motors) and nonlinear model of wheel-rail contact. The vehicle model, with its structure and parameters, corresponds to the electric locomotive Škoda 79E; it has been used previously in $[18,19]$ and compared with experiments reported in [14].

The simulation scenario (designed according to the mentioned experiments) represented start-up of the locomotive from zero speed. A braking six-axle locomotive was coupled to the locomotive to create the load. Pneumatic wheel load equalizers as well as anti-slip protection was disabled, so that the locomotive went into full sliding at low speed. The simulated behaviour included several modes in which the transient rolling character is significant:

- first movement of the vehicle from zero speed,

- change of rolling direction (without change of tangential force direction - experienced when the locomotive was pulled back by the drawgear spring),

- interrupted sliding with the stick-slip effect in the contact,

- initiation and termination of sliding at very low vehicle speed.

As reported in previous publications, the simplified model of transient rolling was used in the simulations - steady rolling could not be used. It has been concluded that the comparison between simulation and experiment cannot be accepted as full validation of the model, since the test conditions and mechanical parameters were not known in the full extent. Nevertheless, the comparison shows that the simulation works correctly in the mentioned "extreme" creepage conditions and is able to show the observed dynamical phenomena, particularly the vibration in the drive system connected with the stick-slip phases of full wheelset sliding. The levels of vibration (in terms of accelerations taken at the axle gearbox) are also comparable.

In the present study, the same simulation is repeated with the STR contact model replaced by the creep force filtering method. The new method is accepted as suitable if it gives results close to the STR model.

It should be noted, however, that the CFFM is not used with the Freibauer/Polách model here. Instead, numerical integration of traction distribution is peformed in the same discretization grid as for the STR. It is obvious that the main advantage of the proposed method is lost; the purpose of this approach is to exclude the effect of discretization error, i.e., the observed differences will include only what is inherent to the treatment of the transient effect itself. 

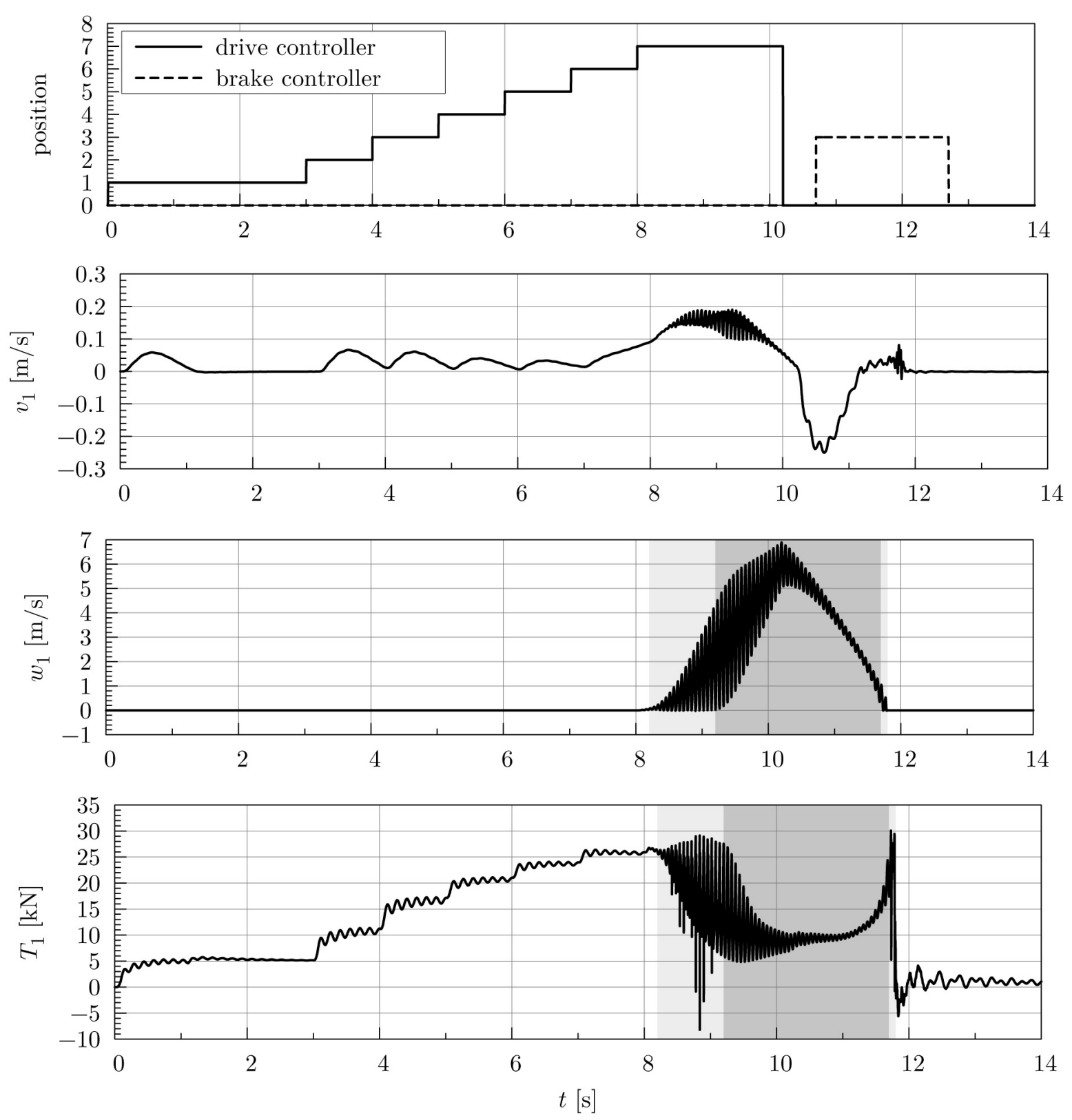

Fig. 8. Simulation results with the CFFM: $v_{1}$ speed of forward motion, $w_{1}$ creep velocity and $T_{1}$ tangential force for the first wheelset. Lighter shaded areas indicate sliding with stick-slip effect, the area with darker shading means uninterrupted sliding

The simulation using CFFM is documented in time history plots in Fig. 8. The first plot shows the input of driving and braking controllers to clarify the stages of the simulation scenario. Further, forward velocity of the first wheelset $v_{1}$ is plotted. Creep velocity $w_{1}$ and tangential force $T_{1}$ of the first wheelset (which is unloaded and starts sliding first) follow. The phases of stick-slip motion and uninterrupted sliding are indicated in the plots. The results with STR are not shown for comparison as little difference would be seen at this scale.

To compare the results of the simulations using STR and CFFM, tangential force $T_{1}$ is plotted in Fig. 9 with the time span limited to 8.0-13.0 s. From this comparison, it may be concluded that the function of the new proposed method is correct and no significant differences from the more complicated model are seen in the simulation results. 


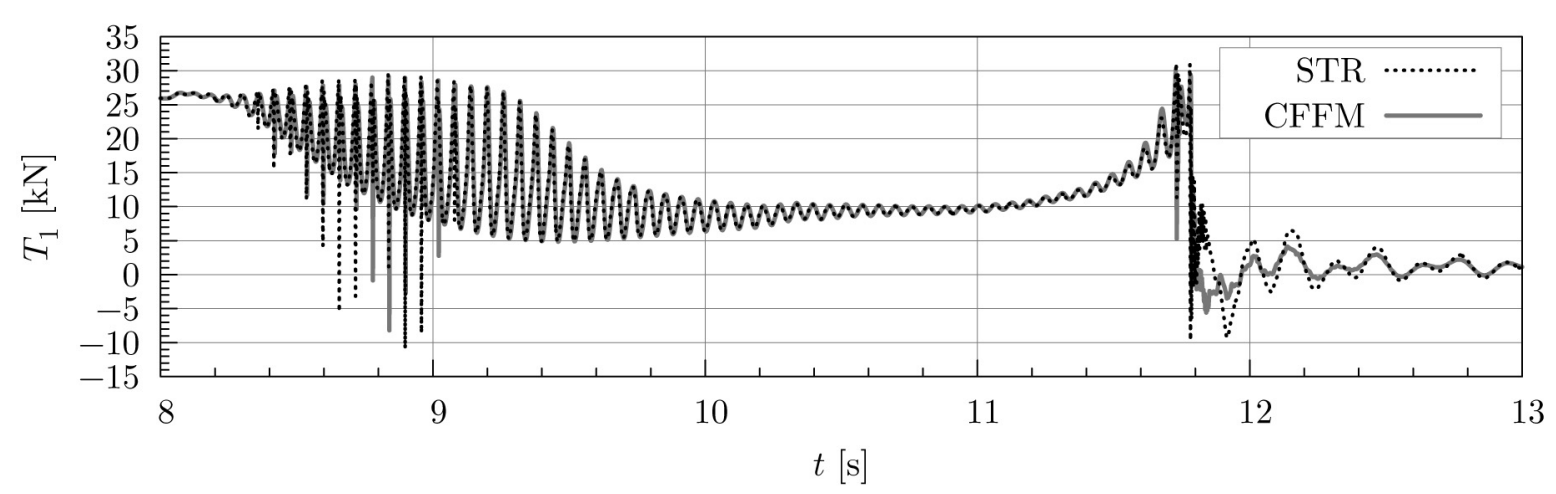

Fig. 9. Time histories of tangential force for STR and CFFM compared

\section{Conclusion}

In this paper, an explanation of the transient rolling contact problem was given. It was stated that for many problems of rail vehicle dynamics, transient rolling is of little importance, whereas there are still some cases where it must not be ignored. The calculation of traction force of a locomotive at very low speed is one of them.

To allow numerical simulation for this case, simplified model of transient rolling was previously developed. It performs numerical calculation and integration of traction distribution in each simulation step. In the present paper, another approach to transient rolling contact was proposed. It can be characterized as approximative and "quasi-transient", not intended for the study of the transient phenomena themselves, but faster in calculation and, especially, easier to use in a program code.

The new approach consists in using a steady-state contact model (Freibauer/Polách) and smoothing the output creep force with a filter with suitably determined parameters. Thence it is called creep force filtering method, CFFM. Its principle as well as guidance to implementation in simulation code has been given.

The CFFM was first tested outside of dynamic simulations, on the basis of input creepage sequences. An example with step increase in creepage is documented here. There is difference between the results of STR and CFFM, however extending the comparison by the Kalker's exact theory leads to a positive conclusion: the result of CFFM closely follows the exact solution. Further, the new method was included in dynamic simulation of a locomotive starting from zero speed and reaching the adhesion limit. Little difference between STR and CFFM was observed in the results. Therefore it may be concluded that the proposed creep force filtering method provides a suitable solution for simulations where a steady rolling model would produce incorrect results.

\section{Acknowledgements}

The research was supported by Competence Center of Rail Vehicles, project No. TE01020038, Technology Agency of the Czech Republic. 


\section{References}

[1] Alonso, A., Giménez, J. G., Non-steady state modelling of wheel-rail contact problem for the dynamic simulation of railway vehicles, Vehicle System Dynamics 46 (3) (2008) 179-196. https://doi.org/10.1080/00423110701248011

[2] Bruni, S., Vinolas, J., Berg, M., Polach, O., Stichel, S., Modelling of suspension components in a rail vehicle dynamics context, Vehicle System Dynamics 49 (7) (2011) 1021-1072. https://doi.org/10.1080/00423114.2011.586430

[3] CONTACT: Vollebregt \& Kalker's rolling and sliding contact model (online), URL: http://kalkersoftware.org, last update August 2016.

[4] Čáp, J., Theoretical analysis of tangential and interactive forces in wheel-rail contact, Scientific Papers of the University of Pardubice, Ser. B 5 (1999) 29-48. (in Czech)

[5] Freibauer, L., Adhesion of a vehicle wheel on a track, In: 7. vedecká konferencia VŠDS v Žiline Zborník referátov, Ž, VŠDS v Žiline, 1983, pp. 214-219. (in Czech)

[6] Freibauer, L., Rus, L., Zahrádka, J., Rail vehicle dynamics, NADAS, Praha, 1991. (in Czech)

[7] Guiral, A., Alonso, A., Baeza, L., Giménez, J. G., Non-steady state modelling of wheel-rail contact problem, Vehicle System Dynamics 51 (1) 91-108. https://doi.org/10.1080/00423114.2012.713499

[8] Hertz, H., On the contact of elastic solids, Journal für die reine und angewandte Mathematik 92 (1882) 156-171. (in German)

[9] Iwnicki, S., Handbook of railway vehicle dynamics, CRC/Taylor \& Francis, 2006.

[10] Johnson, K. L., Contact mechanics, Cambridge University Press, Cambridge, 2003.

[11] Kalker, J. J., Simplified theory of rolling contact, Delft Progress Report, Series C 1 (1973) 1-10.

[12] Kalker, J. J., Survey of wheel-rail rolling contact theory, Vehicle System Dynamics 5 (1979) 317-358. https://doi.org/10.1080/00423117908968610

[13] Kalker, J. J., Three-dimensional elastic bodies in rolling contact, Kluwer Academic Publishers, Dordrecht, 1990. https://doi.org/10.1007/978-94-015-7889-9

[14] Lata, M., Voltr, P., Identification of transient phenomena occurring an the initiation and termination of wheelset sliding, Transport 27 (1) (2012) 86-91. https://doi.org/10.3846/16484142.2012.668498

[15] Polách, O., Creep forces in simulations of traction vehicles running on adhesion limit, Wear 258 (2005) 992-1000. https://doi.org/10.1016/j.wear.2004.03.046

[16] Spiryagin, M., Cole, C., Sun, Y. Q., McClanachan, M., Spiryagin, V., McSweeney, T., Design and simulation of rail vehicles, CRC Press, 2014. https://doi.org/10.1201/b17029

[17] Vollebregt, E. A. H., Iwnicki, S., Xie, G., Shackleton, P., Assessing the accuracy of different simplified frictional rolling contact algorithms, Vehicle System Dynamics 50 (1) (2012) 1-16. https://doi.org/10.1080/00423114.2011.552618

[18] Voltr, P., Lata, M., Dynamical behaviour of a locomotive drive system at the adhesion limit experiment and simulation, Proceedings of the 22nd International Symposium on Dynamics of Vehicles on Roads and Tracks, Manchester Metropolitan University, Manchester, 2011.

[19] Voltr, P., Lata, M., Transient problems in rolling contact of wheel and rail, Proceedings of the 9th International Conference on Railway Bogies and Running Gears, Budapest University of Technology, Budapest, 2014, pp. 145-152. 